\title{
DUAL STATE-PARAMETER UPDATING SCHEME ON A CONCEPTUAL HYDROLOGIC MODEL USING SEQUENTIAL MONTE CARLO FILTERS
}

\author{
Seong Jin $\mathrm{NOH}^{1}$, Yasuto TACHIKAWA ${ }^{2}$, Michiharu SHIIBA ${ }^{3}$ and Sunmin $\mathrm{KIM}^{4}$ \\ ${ }^{1}$ Student Member of JSCE, Graduate Student, Dept. of Urban and Env. Eng., Kyoto University \\ (Nishikyo-ku, Kyoto 615-8540, Japan) E-mail:seongjin.noh@gmail.com \\ ${ }^{2}$ Member of JSCE, Dr. Eng., Associate Professor, Dept. of Civil and Earth Resources Eng., Kyoto University \\ (Nishikyo-ku, Kyoto 615-8540, Japan) \\ ${ }^{3}$ Member of JSCE, Dr. Eng., Professor, Dept. of Civil and Earth Resources Eng., Kyoto University \\ (Nishikyo-ku, Kyoto 615-8540, Japan) \\ ${ }^{4}$ Member of JSCE, Dr. Eng., Lecturer, Dept. of Civil and Earth Resources Eng., Kyoto University \\ (Nishikyo-ku, Kyoto 615-8540, Japan)
}

\begin{abstract}
Applications of data assimilation techniques have been widely used to improve upon the predictability of hydrologic modeling. Among various data assimilation techniques, sequential Monte Carlo (SMC) filters, known as "particle filters", provide the capability to handle non-linear and non-Gaussian state-space models. This paper proposes a dual state-parameter updating scheme (DUS) based on SMC methods to estimate both state and parameter variables of a hydrologic model. We introduce a kernel smoothing method for the robust estimation of uncertain model parameters in the DUS. The applicability of the dual updating scheme is illustrated using the implementation of the storage function model on a middle-sized Japanese catchment. We also compare performance results of DUS combined with various SMC methods, such as SIR, ASIR and RPF.
\end{abstract}

Key Words: Data assimilation, sequential Monte Carlo, particle filter, storage function model

\section{INTRODUCTION}

Identification and minimization of uncertainty are key issues in the hydrologic prediction. Data assimilation is a way to integrate information from a variety of sources to improve model accuracy, considering the uncertainty in both the measurement and modeling system. Among data assimilation techniques, the sequential Monte Carlo (SMC) methods are a Bayesian learning process in which the propagation of all uncertainties is carried out by a suitable selection of randomly generated particles without any assumptions about the nature of the distributions. Unlike the various Kalman filter-based methods that are basically limited to the linear system equation and the assumption of Gaussian distribution errors, SMC filters have the advantage of being applicable to non-linear, non-Gaussian, state-space models. Since their introduction in $1993^{1)}$, the application of these powerful and versatile methods has been increasing in various areas, including pattern recognition, target tracking, financial analysis, and robotics. Only in recent years has the application of these methods been included in hydrology research ${ }^{2), 3,4)}$.

In the practical use of hydrologic models, estimated states are highly sensitive to the uncertainty of model parameters. Furthermore, there is no guarantee that parameters calibrated from previous data are the optimum in the current prediction. Therefore, updating state variables based on inappropriate parameters will likely increase uncertainty in the forecasting of hydrologic models. In this respect, sequential estimates of the parameters and state variables are needed to enable the model to generate accurate forecasts.

In this paper, we propose a dual state-parameter updating scheme (DUS) based on the SMC filters for the estimation of both the state and parameter variables of a hydrologic model. A kernel smoothing method is introduced for the robust estimation of uncertain model parameters in the 
DUS. We illustrate its applicability for hydrologic forecasting on a middle-sized Japanese catchment using a conceptual hydrologic model.

The paper is organized in the following way. Section 2 outlines the Bayesian filtering theory; the sequential Monte Carlo filters, known as particle filters, which are based on the sequential importance sampling (SIS); and parameter inference approaches in SMC. In Section 3, the case study demonstrating the applicability of the SMC filters is presented. The SMC filters are applied for real-time forecasting of river discharge of the Katsura River catchment using the storage function (SF) model. Sequential data assimilation is performed by two different schemes via the SMC filters: state only updating and dual state-parameter updating. Comparisons of the performance results of various SMC filters are presented. Section 4 summarizes the methodology and the analysis results.

\section{METHODOLOGY}

\section{(1) Bayesian filtering theory}

To define the problem of the Bayesian filtering, consider a generic dynamic state-space model which can be described as follows:

$$
\begin{aligned}
x_{t}=f\left(x_{t-1}, \theta, u_{t}\right)+\omega_{t} & \omega_{t} & \sim N\left(0, W_{t}\right) \\
y_{t}=h\left(x_{t}, \theta^{\prime}\right)+v_{t} & v_{t} & \sim N\left(0, V_{t}\right)
\end{aligned}
$$

where $x_{t} \in \mathfrak{R}^{n_{x}}$ is the $n_{x}$ dimensional vector denoting the system state at time $t$. The operator $f: \Re^{n_{x}} \rightarrow \mathfrak{R}^{n_{x}}$ expresses the system transition in response to the forcing data $u_{t}$, and parameters $\theta$. $h: \mathfrak{R}^{n_{x}} \rightarrow \mathfrak{R}^{n_{x}}$ expresses the measurement function. $\omega_{t}$ and $v_{t}$ represent the model and the measurement error, and $W_{t}$ and $V_{t}$ represent the covariance of the error, respectively. In particular, we seek filtered estimates of $x_{t}$ based on the set of all available measurements $y_{1: t}=\left\{y_{i}, i=1, \ldots, t\right\}$. In the Bayesian recursive estimation, if the system and measurement models are non-linear and non-Gaussian, it is not possible to construct the posterior probability density function (PDF) of the current state $x_{t}$ given all the measurement analytically. When the analytic solution is intractable, an optimal solution can be approximated by the SMC filters explained in the next section.

\section{(2) Sequential importance sampling (SIS)}

Sequential Monte Carlo (SMC) filters are a set of simulation-based methods that provide a flexible approach to computing the posterior distribution without any assumptions about the nature of the distributions. The key idea of SMC is based on point mass ("particle") representations of probability densities with associated weights as ${ }^{5)}$ :

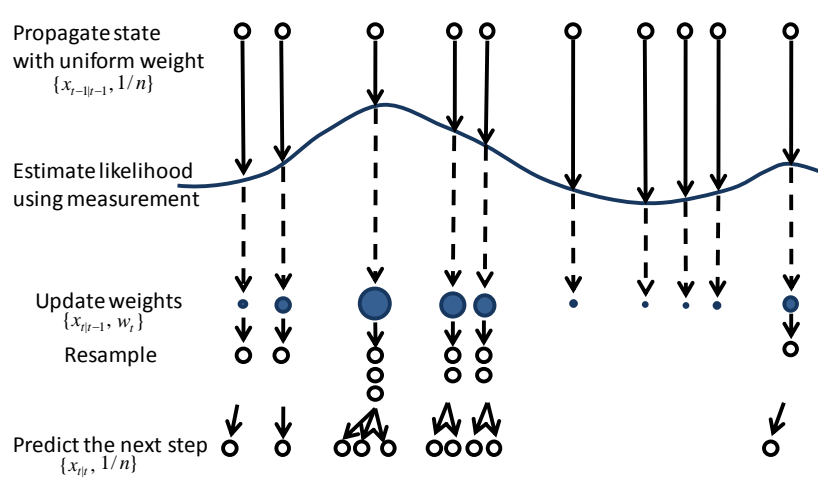

Fig. 1 A single cycle of SMC.

$$
p\left(x_{t} \mid y_{1: t}\right) \approx \sum_{i=1}^{n} w_{t}^{i} \delta\left(x_{t}-x_{t}^{i}\right)
$$

where $x_{t}^{i}$ and $w_{t}^{i}$ denote the $i^{\text {th }}$ posterior state ("particle") and its weight, respectively, and $\delta(\cdot)$ denotes the Dirac delta function.

Since it is usually impossible to sample from the true posterior PDF, an alternative is to sample from a proposal distribution, also called importance density, denoted by $q\left(x_{t} \mid y_{t}\right)$. After the several steps of computation, the recursive weight updating can be derived as follows:

$$
w_{t}^{i} \propto w_{t-1}^{i} \frac{p\left(y_{t} \mid x_{t}^{i}\right) p\left(x_{t}^{i} \mid x_{t-1}^{i}\right)}{q\left(x_{t}^{i} \mid x_{t-1}^{i}, y_{t}\right)}
$$

The choice of importance density is one of the most critical issues in the design of SMC filters ${ }^{6}$. The most popular choice is the transitional prior as

$$
q\left(x_{t}^{i} \mid x_{t-1}^{i}, y_{t}\right)=p\left(x_{t}^{i} \mid x_{t-1}^{i}\right)
$$

By substituting Eq. (5) into Eq. (4), the weight updating becomes

$$
w_{t}^{i} \propto w_{t-1}^{i} p\left(y_{t} \mid x_{t}^{i}\right)
$$

With these particles and associated weights, the estimated state vector $\hat{x}_{t}$ is the weighted mean of particles as:

$$
\hat{x}_{t}=\sum_{i=1}^{n} w_{t}^{i} x_{t \mid t-1}^{i}
$$

The SIS algorithm is a Monte Carlo method that forms the basis for most SMC filters. A common problem with the SIS algorithm is the degeneracy phenomenon, where after a few iterations, all but one particle will have negligible weight. The degeneracy phenomenon can be reduced by performing the resampling step whenever a significant degeneracy is observed. A graphical representation of SMC is illustrated in Fig. 1. At the top we start with a uniformly weighted random measure. Then we use the received measure $y_{t}$ to compute its importance weight of each particle. If necessary, a resampling step is executed to select important particles with a uniform weight. If the number of particles is $n$, the weight is $1 / n$. The last step is a prediction introducing process noise. 


\section{(3) Variant of SMC filters}

Several variants of SMC filters have been proposed in the literature to overcome the degeneracy and sample impoverishment and to improve selection of importance density. The sample importance resampling (SIR) filter is derived from the SIS algorithm by performing the resampling step at every time index. The auxiliary SIR (ASIR) filter performs the resampling step at the previous time step, attempting to mimic the optimal importance density. The regularized particle filter (RPF) was suggested as a method to improve the sample diversity. It is worth noting that these filters can be (and often are) combined ${ }^{6)}$.

\section{(4) Parameter inference}

Identification of parameter uncertainty is essential to obtain unbiased data assimilation. To handle inference of the unknown parameters, the concept of "artificial evolution" can be applied. That means that the parameter vector $\theta$ is fluctuated at each time step, adding an independent, zero-mean normal increment as follows:

$$
\theta_{t}=\theta_{t-1}+\zeta_{t} \quad \zeta_{t} \sim N\left(0, s^{2} V_{t-1}^{\theta}\right)
$$

where $\zeta_{t}$ is random noise, $V_{t-1}^{\theta}$ is the variance of parameter particles at time $t-1$ before resampling, and $s$ is a small tuning parameter. The drawback of this approach is that estimated posterior distribution of parameters becomes more diffuse compared to the actual ones ${ }^{7)}$. Kernel smoothing ${ }^{8)}$ is one remedy for this problem and is accomplished by determining the covariance of parameters based on particles from previous time points. The smooth kernel density can be a mixture of Gaussian densities as follows:

$$
P\left(\theta_{t} \mid y_{1}, \ldots, y_{t-1}\right) \sim \sum_{i=1}^{n} w_{t-1}^{i} N\left(\theta_{t} \mid m_{t-1}^{i}, h^{2} V_{t-1}^{\theta}\right)
$$

where $h$ is the variance reduction parameter. The kernel locations $m_{t-1}^{i}$ are specified by a shrinkage rule forcing the particles to be closer to their mean:

$$
m_{t-1}^{i}=a \theta_{t-1}^{i}+(1-a) \bar{\theta}_{t-1} \text { with } a=\sqrt{1-h^{2}}
$$

It can be verified that the mixture probability in Eq. (9) has a covariance matrix $V_{t-1}^{\theta}$ and that it does not increase over time ${ }^{8)}$. A dual state-parameter updating scheme with kernel smoothing via the SIR particle filter can be summarized in Fig. 2.

\section{IMPLEMENTATION}

\section{(1) Study area}

The SMC filters were applied to the Katsura River catchment (Fig. 3) to improve the river flow forecasting. This catchment is located in Kyoto,

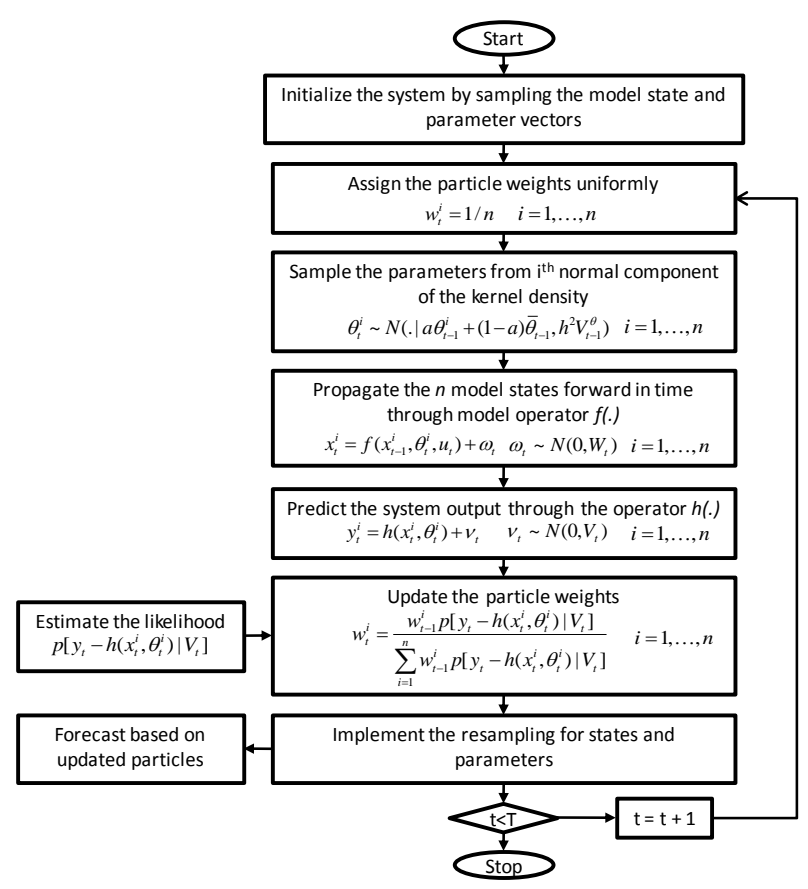

Fig. 2 Flowchart of the dual state-parameter updating scheme with kernel smoothing via the SIR particle filter.

Japan, and covers an area of $1,100 \mathrm{~km}^{2}\left(887 \mathrm{~km}^{2}\right.$ at the Katsura station). There are 13 rainfall observation stations and 6 river flow observation stations. The Hiyoshi dam is located upstream, and the outflow record from that reservoir has been considered to be input data in a hydrologic model.

\section{(2) Hydrological model and simulation condition}

The storage function (SF) model ${ }^{9)}$ is one of the most commonly used conceptual hydrologic models for flood prediction due to its simple numerical procedure and its proper regeneration of nonlinear characteristics of flood runoff. The state-space form of the SF model adapted in this catchment is as follows:

$$
\begin{gathered}
\frac{d s(t)}{d t}=r_{e}\left(t-T_{L}\right)-\left(\frac{s(t)}{k}\right)^{1 / p}+\omega_{t} \\
q_{\text {sim }}(t)=\frac{A_{\text {down }}\left(\frac{s(t)}{3.6}\right)^{1 / p}+q_{\text {dam }}\left(t-T_{\text {dam }}\right)}{} \\
q_{t}=q_{\text {sim }}(t)+v_{t}
\end{gathered}
$$

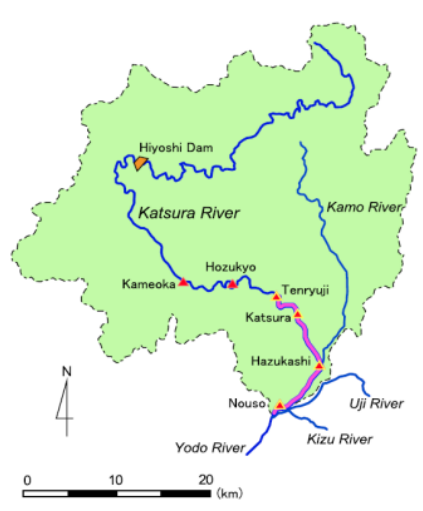

Fig. 3 The Katsura River catchment. 
where $s$ is catchment storage $(\mathrm{mm}), t$ is time $(\mathrm{hr})$, $A_{\text {down }}$ is the downstream area from the dam $\left(\mathrm{km}^{2}\right)$, $q_{\text {sim }}$ is simulated river discharge $\left(\mathrm{m}^{3} / \mathrm{s}\right), q_{t}$ and $q_{\text {dam }}$ are observed discharge at the Katsura gauging station and at the Hiyoshi dam $\left(\mathrm{m}^{3} / \mathrm{s}\right), T_{L}$ and $T_{\text {dam }}$ are the lag time parameters of catchment and outflow from the dam reservoir (hr), and $k$ and $p$ are model parameters. $\omega_{t}$ and $v_{t}$ are the state and the measurement error, respectively. Effective rainfall $r_{e}$ is estimated as follows:

$$
r_{e}=r \times f^{*} \text { with } f^{*}= \begin{cases}1 & r_{\text {accum }} \geq R_{s a} \\ f & r_{\text {accum }}<R_{s a}\end{cases}
$$

where $r$ is rainfall $(\mathrm{mm} / \mathrm{hr}), f$ is the runoff coefficient, $r_{\text {accum }}$ is the accumulated rainfall amount $(\mathrm{mm})$, and $R_{s a}$ is the saturation amount $(\mathrm{mm})$. Areal mean values of hourly observed rainfall from the 13 gauging stations were used as model input. Six model parameters, including $k, p, T_{L}, T_{\text {dam }}, f$ and $R_{s a}$, have been estimated from the events of 2004. In the state only updating scheme, pre-calibrated parameter values were used. On the other hand, the dual state-parameter updating scheme has been performed on five model parameters, excluding $T_{\text {dam }}$, which showed stable values compared to others. Both simulations were performed by the SIR particle filter with 3,000 particles. Covariance of the error of system $\left(W_{t}\right)$ and measurement $\left(V_{t}\right)$ were assumed to be $4 \mathrm{~mm}$ and $10 \%$ of the current observed discharge, respectively.
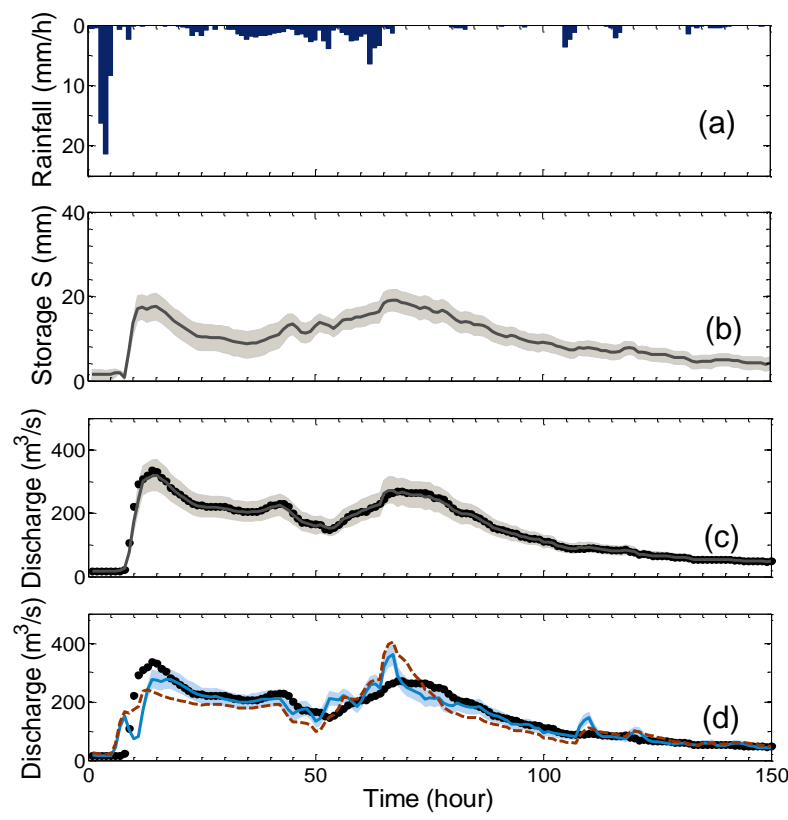

Fig. 4 Results of the state only updating via the SIR particle filter from 11 to 16 July 2007. (a) Hourly precipitation. (b) Catchment storage. (c) Updated river discharge. (d) 3-hour-lead forecasted river discharge. Black dots represent observed discharge. Blue line and area represent mean value and $95 \%$ confidential interval, respectively. Dashed line represents a deterministic modeling case.

\section{(3) State only updating scheme}

Fig. 4 shows the simulation results of the state only updating via the SIR particle filter compared to observations and a deterministic prediction. In this scheme, particles are resampled in each observation time step, and catchment storage $(s)$ is fluctuated according to the system noise.

While updated river discharge using a state only updating scheme shows good conformity between observation and simulation (Fig. 4(c)), a forecast based on the same particles does not reproduce the river flow properly compared to a deterministic prediction (Fig. 4(d)). To compare off-line optimal parameters with those calibrated from the past event (Table 1), several parameters show quite different values. In this respect, it can be inferred that state updating based on inappropriate parameters may be one of the causes misleading the forecast.

\section{(4) Dual state-parameter updating scheme}

In the dual state-parameter updating scheme, initial values of each parameter have been set to uniform distribution with widths that cover deviations of pre-calibrated parameter distributions. In other words, true static values of parameters are assumed to be located within these initial distributions. Inference of five parameters (e.g., $k$, $p, T_{L}, f$ and $R_{s a}$ ) was performed by the kernel smoothing method in DUS. The value of kernel smoothing parameter $a$ in Eq. (9) was set as 0.95.
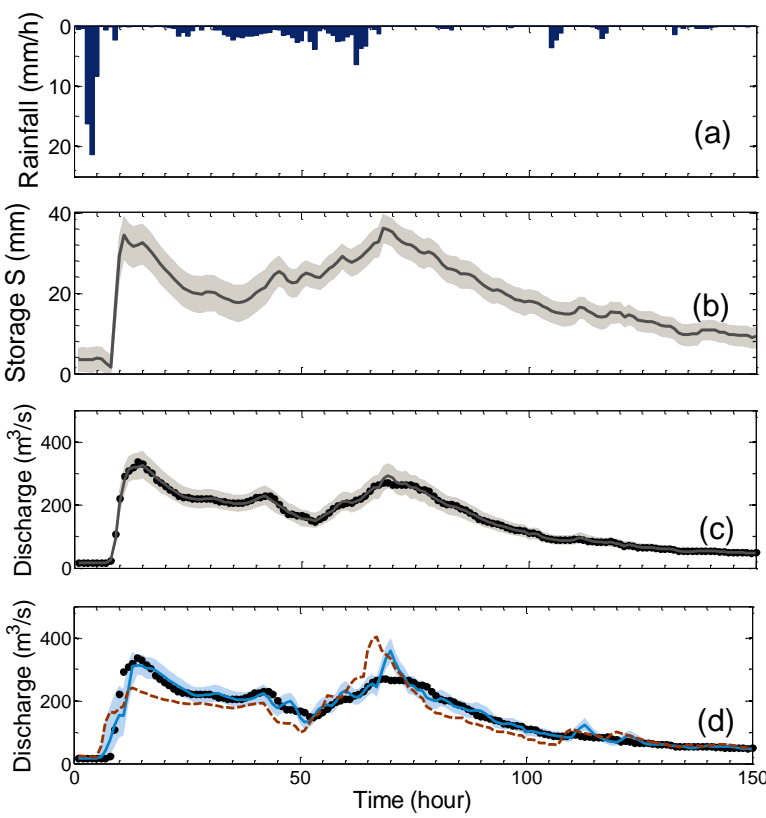

Fig. 5 Results of the dual state-parameter updating via the SIR particle filter from 11 to 16 July 2007. (a) Hourly precipitation. (b) Catchment storage. (c) Updated river discharge. (d) 3-hour-lead forecasted river discharge. Black dots represent observed discharge. Blue line and area represent mean value and $95 \%$ confidential interval, respectively. Dashed line represents a deterministic modeling case. 
Table 1. Parameter information.

\begin{tabular}{|c|c|c|c|}
\hline $\begin{array}{c}\text { Model } \\
\text { parameters }\end{array}$ & $\begin{array}{c}\text { Pre-calibrated } \\
\text { from 2004 events }\end{array}$ & $\begin{array}{c}\text { Off-line optimum } \\
\text { from 2007 events }\end{array}$ & $\begin{array}{c}\text { Initial range } \\
\text { for dual updating }\end{array}$ \\
\hline \hline$k(-)$ & 17.0 & 30.0 & $10.0 \sim 40.0$ \\
\hline$p(-)$ & 0.6 & 0.66 & $0.4 \sim 0.9$ \\
\hline$T_{L}(\mathrm{hr})$ & 3.8 & 6.0 & $3.0 \sim 7.0$ \\
\hline$f(-)$ & 0.33 & 0.65 & $0.1 \sim 0.8$ \\
\hline$R_{s a}(\mathrm{~mm})$ & 82.0 & 105.0 & $50.0 \sim 150.0$ \\
\hline$T_{\text {dam }}(\mathrm{hr})$ & 4.0 & 4.0 & 4.0 \\
\hline
\end{tabular}

Fig. 5 illustrates the simulation results of the dual state-parameter updating. Compared with the state only updating case, a forecast by the dual updating scheme shows better conformity with observations (Fig. 5(d)). Furthermore, the unexpected drawdown of hydrograph in the rising part (Fig. 4(d)) is not shown in the dual updating case. Traces of the catchment storage $s$ present different patterns in Fig. 4(b) and Fig. 5(b), whereas updated discharge hydrographs show similar traces in both cases.

Fig. 6 presents the traces of parameter distribution. One can observe a significant reduction of parameter uncertainty for all parameters after the first flood peak. In comparison with the off-line optimum (Table 1), estimated parameters show similar ranges, especially in parameter $k, T_{L}$ and $f$.

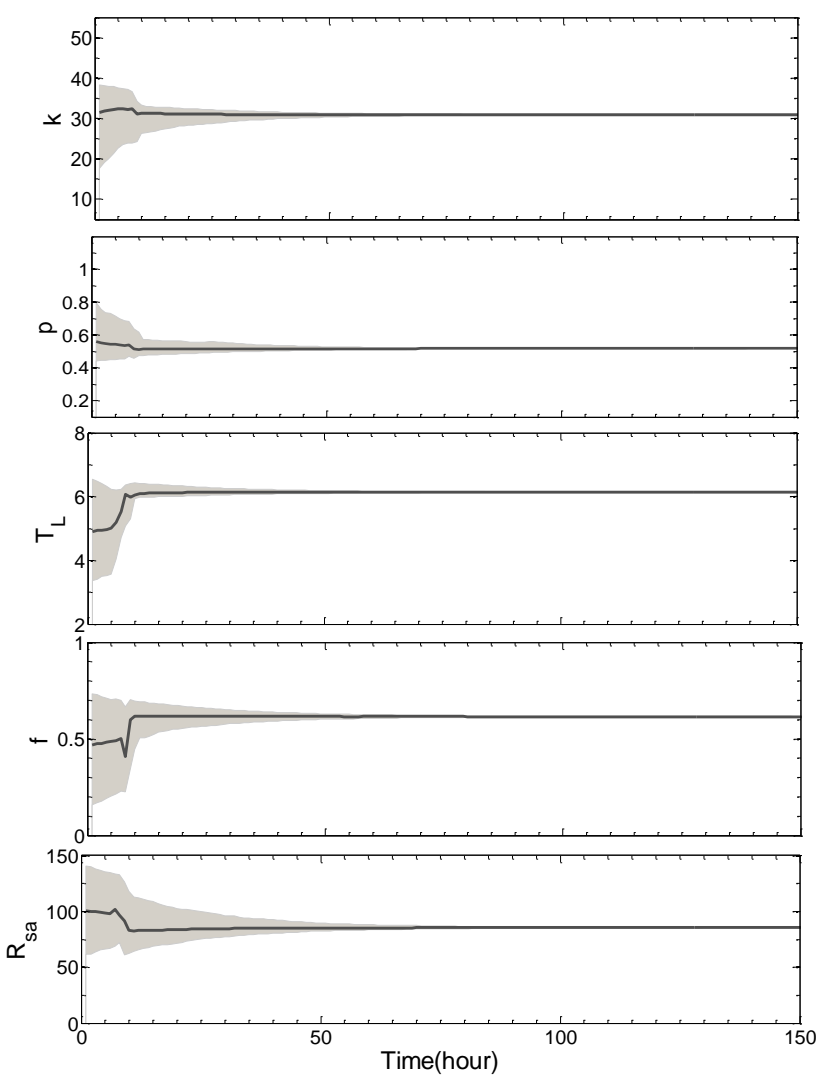

Fig. 6 Traces of parameter $k, P, T_{L}, f, R_{s a}$ of the SF model using dual state-parameter updating of the SIR particle filter from 11 to 16 July 2007. Black lines represent median value, and gray area represents $95 \%$ confidential interval.

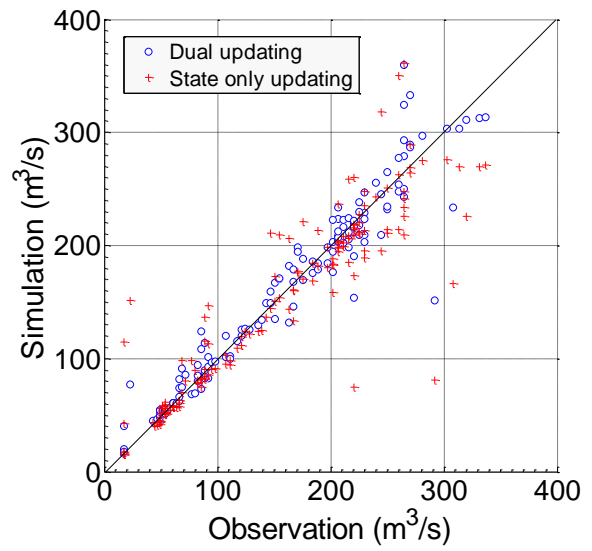

Fig. 7 Scatter diagram of simulation results. Cross dots represent results of state only updating. Circle dots represent results of dual state-parameter updating.

Table 2. Statistics on model accuracy.

\begin{tabular}{|c|c|c|c|}
\hline & Deterministic & $\begin{array}{c}\text { State only } \\
\text { updating }\end{array}$ & Dual updating \\
\hline \hline RMSE $\left(\mathrm{m}^{3} / \mathrm{s}\right)$ & 44.6 & 36.4 & 20.4 \\
\hline $\begin{array}{c}\text { Nash } \\
\text {-Sutcliffe }\end{array}$ & 0.73 & 0.82 & 0.94 \\
\hline
\end{tabular}

It is worth noting that when the artificial evolution is applied for parameter inference instead of kernel smoothing in the dual updating scheme, estimated parameters present more diffusive distributions and unstable inference is produced resulting in different posterior distributions at each simulation. However, inference from the kernel smoothing presents relatively consistent results because there is less uncertainty of parameters.

In the scatter diagram shown in Fig. 7, the dual state-parameter updating scheme presents enhanced simulation results in the overall flow regime from high flow to low flow. Additionally, the model accuracy criteria shown in Table 2 confirm that the DUS is superior to other simulations.

\section{(5) Comparison of various SMC filters}

Several different versions of the SMC filters, such as SIR, ASIR, and RPF with the MCMC move step, were implemented under the same simulation conditions. The Markov chain Monte Carlo (MCMC) move step of RPF, which is used for improving sample diversity in the resampling step, is based on the Metropolis-Hastings algorithm ${ }^{10}$. The dual state-parameter updating scheme has been adapted in all the cases with 3,000 particles. A comparison of the simulated discharge hydrograph is illustrated in Fig. 8. There is no significant difference in the estimated 3-hour-ahead forecasting via three SMC filters.

Although three SMC filters reproduce river discharge properly in the first flood peak (1 30 hour) and the recession part, all the SMC methods 


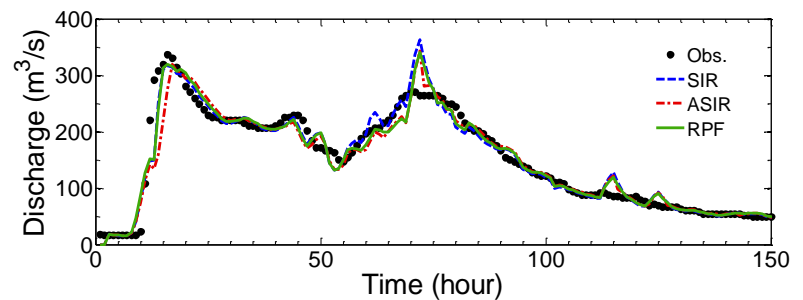

Fig. 8 Forecasted river discharge (3 hour ahead) by three SMC filters from 11 to 16 July 2007. Black dots represent observed discharge.
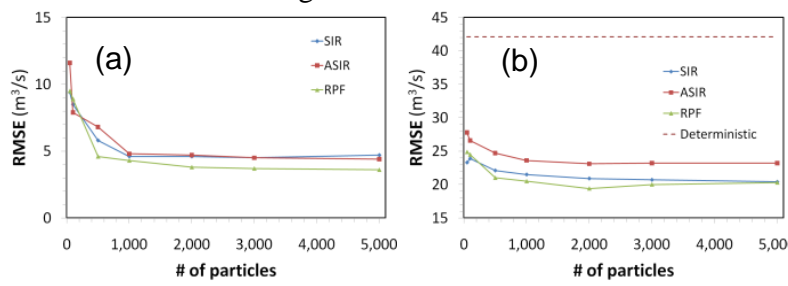

Fig. 9 Sensitivity analysis of the effects of particle numbers on the prediction accuracy. (a) Updated river discharge. (b) Forecasted river discharge.

overestimate the discharge during 65-80 hours. Uncertainty of forcing data (rainfall) and no consideration of spatial heterogeneity in the SF model are plausible reasons.

Sensitivity analysis was performed concerning the effects of particle numbers on the prediction accuracy (Fig. 9). RMSE statistics of simulated discharge show stabilized accuracy in both updating and forecasting via three SMC filters when the number of particles exceeds 1,000. In terms of forecasting accuracy, SIR and RPF show similar RMSE statistics, while ASIR presents a slightly higher number of errors than others. Researchers also stated in a previous study ${ }^{6}$ that if the importance density of ASIR does not characterize the transitional prior $p\left(x_{t} \mid x_{t-1}^{i}\right)$ for some reason (e.g., process noise is large), the use of ASIR can even degrade the performance. The simulation time for 1,000 particles is less than $2 \mathrm{~min}$ in three SMC filters, which is short enough to be applied for real-time forecasting.

\section{CONCLUSIONS}

Sequential Monte Carlo (SMC) filters were applied to a conceptual hydrologic model, the storage function model, using state only updating and the dual state-parameter updating scheme. The river discharge forecast via the SMC filters was compared with observations. The forecast provided by the dual state-parameter updating scheme was superior to that of state only updating and deterministic modeling in terms of the model accuracy criteria, a scatter diagram, and simulated hydrographs. In the dual state-parameter updating scheme, parameter inference was performed by the kernel smoothing method. A significant reduction of parameter uncertainty was observed for all parameters after the first flood peak, and estimated parameter distributions showed good conformity with off-line optimum. Performance results of SIR and RPF showed similar forecasting accuracy, while ASIR resulted in a slightly higher number of errors than others. However, RMSE statistics of three SMC filters presented stable results when the number of particles was over 1,000.

The SMC filters are applicable to more complex hydrologic models, such as process-based and spatially distributed hydrologic models, in which it is difficult to use the conventional data assimilation methods. We will examine the performance of the SMC filters on a distributed hydrologic model.

\section{REFERENCES}

1) Gordon, N. J., Salmond, D. J. and Smith, A. F. M., Novel approach to nonlinear/non-Gaussian Bayesian state estimation, Proc. Inst. Electr. Eng., Vol.140(2), pp.107-113, 1993.

2) Moradkhani, H., Hsu, K. L., Gupta, H. and Sorooshian, S., Uncertainty assessment of hydrologic model states and parameters: Sequential data assimilation using the particle filter, Water Resour. Res., Vol.41, W05012, doi:10.1029/ 2004WR003604, 2005.

3) Smith, P. J., Beven, K. J. and Tawn, J. A., Detection of structural inadequacy in process-based hydrological models: A particle-filtering approach. Water Resour. Res., Vol.44, W01410, doi:10.1029/2006WR005205, 2008.

4) Salaman, P. and Feyen, L., Assessing parameter, precipitation, and predictive uncertainty in a distributed hydrological model using sequential data assimilation with the particle filter, J. Hydrol., Vol. 376, pp.428-442, 2009.

5) Arulampalam, M. S., Maskell, S., Gordon, N. and Clapp, T., A tutorial on particle filters for online nonlinear/ non-Gaussian Bayesian tracking, IEEE Trans. Signal Proces., Vol.50(2), pp.174-188, 2002.

6) Ristic, B., Arulampalam, S. and Gordon, N.: Beyond the Kalman filter: Particle filters for tracking applications, Artech House, 2004.

7) Moradkhani, H., Sorooshian, S., Gupta, H. V. and Houser, P. R., Dual state-parameter estimation of hydrological models using ensemble Kalman filter, Adv. Water Resour., Vol.28, pp.135-147, 2005.

8) Liu, J. and West, M., Combined parameter and state estimation in simulation-based filtering, Sequential Monte Carlo in Practice, Doucet, A., de Freitas, N. and Gordon, N. eds., Springer-Verlag, pp.197-223, 2001.

9) Kimura, T.: The flood runoff analysis method by the storage function model, The Public Works Research Institute, Ministry of Construction, Japan, 1961.

10) Robert, C. P. and Casella, G.: Monte Carlo statistical methods, Springer, New York, 1999.

(Received September 30, 2010) 